\section{Cureus}

\title{
A Novel Applicator for Intracavitary Low-Dose- Rate Brachytherapy (LDR) for Carcinoma of the Uterine Cervix: Results of a Single Institutional Trial
}

Nagy Elsayyad ${ }^{1}$, Cristiane Takita ${ }^{2}$, Gail Walker ${ }^{3}$, Aaron Wolfson ${ }^{2}$, Xiaodong Wu ${ }^{4}$, Arnold M. Markoe 2

1. Department of Radiation Oncology, University of Miami Sylvester Cancer Center 2. Department of Radiation Oncology, University of Miami Sylvester Cancer Center 3. University of Miami Sylvester Cancer Center 4. CyberKnife Center of Miami; University of Miami Miller School of Medicine, Miami, FL

$\square$ Corresponding author: Nagy Elsayyad, nelsayyad@gmail.com

Disclosures can be found in Additional Information at the end of the article

\section{Abstract}

Background: We report results of a study assessing the use of a novel applicator to deliver low-dose-rate brachytherapy (LDR) to carcinoma of the uterine cervix. It offers the advantage of simultaneous loading of vaginal "tandem" and "ovoid-equivalent" sources and obviates the need for vaginal packing.

Purpose: To test the hypothesis that the applicator could deliver, reproducibly, the prescribed dose without compromising the rate of loco-regional (LR) tumor control. End points: 1) doses delivered to Pt. A, rectum, bladder and vaginal surface (as defined by ICRU), 2) variability of such doses amongst the study patients, 3) rates of local control and adverse events.

Methods and Materials: Twenty-five patients with histologically-proven carcinoma of the cervix were entered consecutively. The median duration of follow-up was 38 (7-48) months. Summary statistics were calculated for each end point. Incidence of vaginal stenosis, rectovaginal (RV) fistulae, and proctitis were all reported using NCI, CTCAE version 3.0 (December 2003). Cumulative incidence of local and distant failure as well as Kaplan-Meier estimates of recurrence-free and overall survival were calculated.

Results: No patients received a total dose to point A outside the limit of $10 \%$ variability from the intended dose. A 10\% limit above the maximal operator-defined rectal and bladder doses was not exceeded in any patient. LR control rate was 24 out of 25 (96\%). Three study patients (12\%) developed RV (recto-vaginal) fistulae.

Conclusions: The data indicate that although this applicator delivers sufficient dose to Point A with excellent local control of disease, an unexpectedly high rate of RV fistulae was demonstrated that was due to several factors. Further investigations are needed to determine the optimal role of this novel application system for cervical cancer, such as for patients with "minimal" vaginal involvement.

Published 04/18/2010

(C) Copyright 2010

Elsayyad et al. This is an open access article distributed under the terms of the Creative Commons Attribution License CC-BY 3.0., which permits unrestricted use, distribution, and reproduction in any medium, provided the original author and source are credited.
Categories: Obstetrics/Gynecology, Radiation Oncology

Keywords: cervical cancer, brachytherapy, intracavitary, fistulae, cervix, uterus, rv, recto-vaginal, recto-vaginal fistulae

\section{Introduction}

We present the results of a pilot trial, undertaken in a single institution, aiming at assessing the use of a new vaginal applicator to deliver LDR (low dose rate) brachytherapy to carcinoma of the uterine cervix. The applicator was conceived by two of the authors (AW and XW) at the University of Miami [1]. The design of the applicator is an attempt at providing sufficient dose to cervical tumors extending with "minimal" (no more than $5 \mathrm{~mm}$ thickness) involvement of the middle or lower thirds of the vagina in a
How to cite this article

Elsayyad N, Takita C, Walker G, et al. (April 18, 2010) A Novel Applicator for Intracavitary Low-Dose-Rate Brachytherapy (LDR) for Carcinoma of the Uterine Cervix: Results of a Single Institutional Trial. Cureus 2(4): e10. DOI 10.7759/cureus.10 


\section{Cureus}

single application. The general aim of this study was to test the hypothesis that the applicator could deliver in a reproducible fashion the prescribed dose without decreasing loco-regional (LR) disease control or increasing normal tissue damage probabilities.

\section{Materials And Methods}

The applicator is an intracavitary after-loading system developed at the University of Miami Miller School Of Medicine (UM MSM) and described in a previous publication [1]. It was registered with the United States Patent Office (No. 6,641,518) in November 2003 and is manufactured by Bio-Nucleonics, Inc. (Miami, Florida) under the trademark Gynocyte ${ }^{\mathrm{R}}$. It received FDA approval for commercial use in September 2006. However, there has been no commercial dissemination of this applicator pending maturation of patient data. The Gynocyte ${ }^{\mathrm{R}}$ is made of MRI-compatible plastic material with tungstencontaining alloy shielding, placed both anteriorly and posteriorly at its proximal aspect, for dose reduction to bladder and rectum, respectively. This shielding was used in all applications for subjects treated in this study. The device consists of a cylindrical frame into which two removable cartridges are inserted, each constituting a segment of the cross section of the cylinder. Each cartridge houses, at its cephalad end, a receptacle for an "ovoid-equivalent" source to be held at right angles to the long axis of the device. A central slot allows the insertion of a standard tandem, which allows loading of intrauterine as well as longitudinally arranged intra-vaginal sources. In order to prevent overdose at the apex of the vagina in case the vaginal portion of the centrally-located tandem is simultaneously loaded along with the ovoid-equivalent cartridges, the design includes a $2 \mathrm{~cm}$ inert "spacer" at the extreme proximal location of the Gynocyte ${ }^{\mathrm{R}}$ in between the location of the two sources placed into the ovoid-equivalent carriers

Figure 1 contains a schematic representation of the assembled device in-utero and displays the Gynocyte $^{\mathrm{R}}$ components, including the anterior bladder and posterior rectal shields, the ovoid-equivalent cartridges, and one type of associated intra-uterine tandem. The current version of this applicator has a diameter of $3.5 \mathrm{~cm}$ at its apex. The distance between the center of each ovoid-equivalent and the central vertical axis of the device is $0.7 \mathrm{~cm}$. The distance between the ovoid-equivalent centers and the apical surface is $1 \mathrm{~cm}$. The remaining shaft has a fixed diameter of $3.0 \mathrm{~cm}$. At present, there are no commercially available models to accommodate those clinical situations in which the vaginal dimensions are narrower.
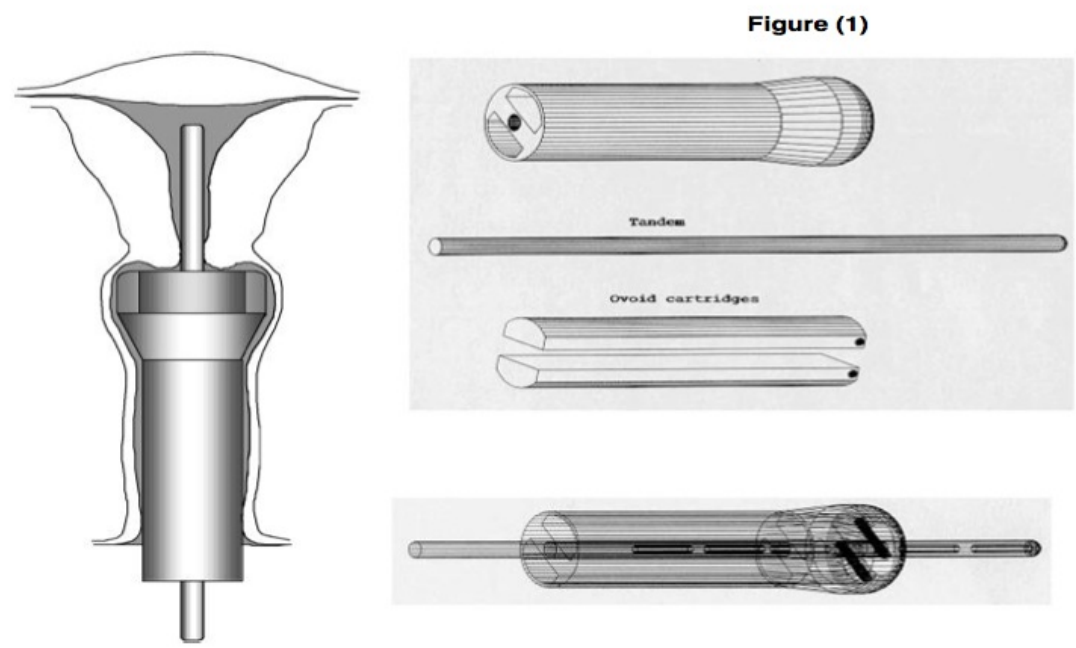

FIGURE 1: Schematic representation of Gynocyte Applicator (BioNucleonics, Inc. of Miami) 


\section{Cureus}

Figure 2 shows the Gynocyte ${ }^{\mathrm{R}}$ and its ovoid-equivalent source carriers with the full complement of tandems as well as the assembled model (with both anterior and posterior tungsten shielding) with a minimally curved tandem in place.
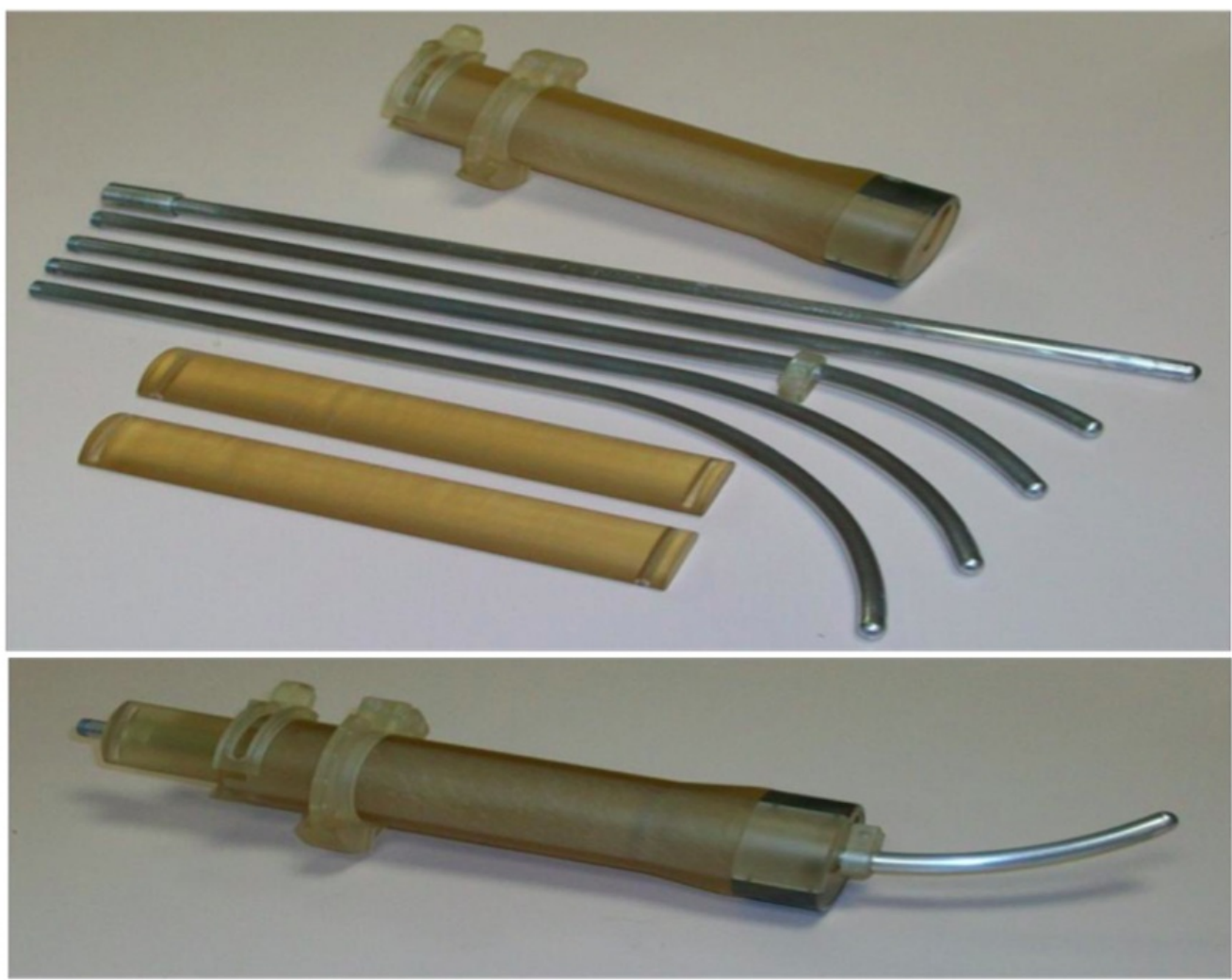

FIGURE 2: Unassembled and assembled Gynocyte Applicator

Figure 3 shows the post-placement orthogonal films. The lateral view clearly indicates the presence of both anterior and posterior shielding of bladder and upper rectum, respectively, along with the injection of rectal contrast permitting the determination of additional rectal points of interest.
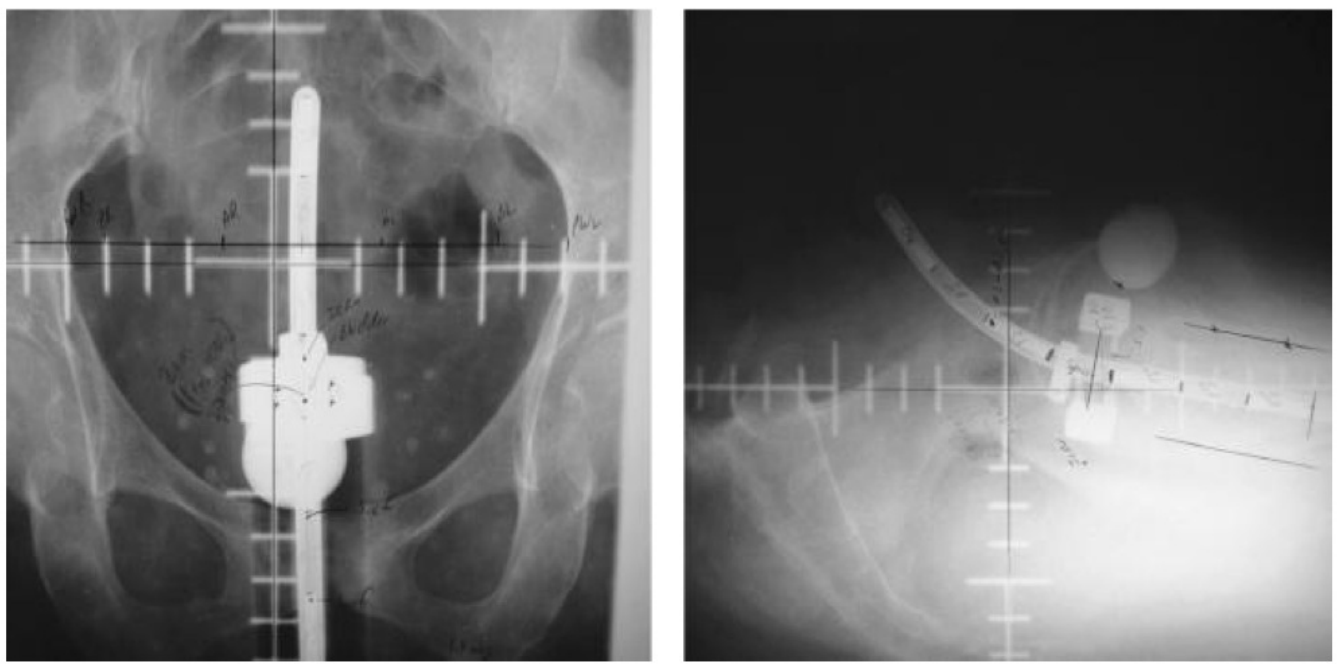

FIGURE 3: Orthogonal films with Gynocyte Applicator in place 
A computer-generated isodose distribution is shown in Figure 4. It is clear from reviewing this figure that the usual pear-shaped isodose distribution depicting point A (as produced by other standard LDR brachytherapy systems commonly used) is preserved with this applicator. Also, one is able to determine not only the ICRU (International Commission on Radiation Units and Measurements) rectal dose-rate but also various other rectal points from the loading of the caudal aspect of the vaginal cylinder portion of the Gynocyte ${ }^{\mathrm{R}}$ with additional Cesium 137 sources that was clinically indicated for this particular patient with "minimal" vaginal disease extension.

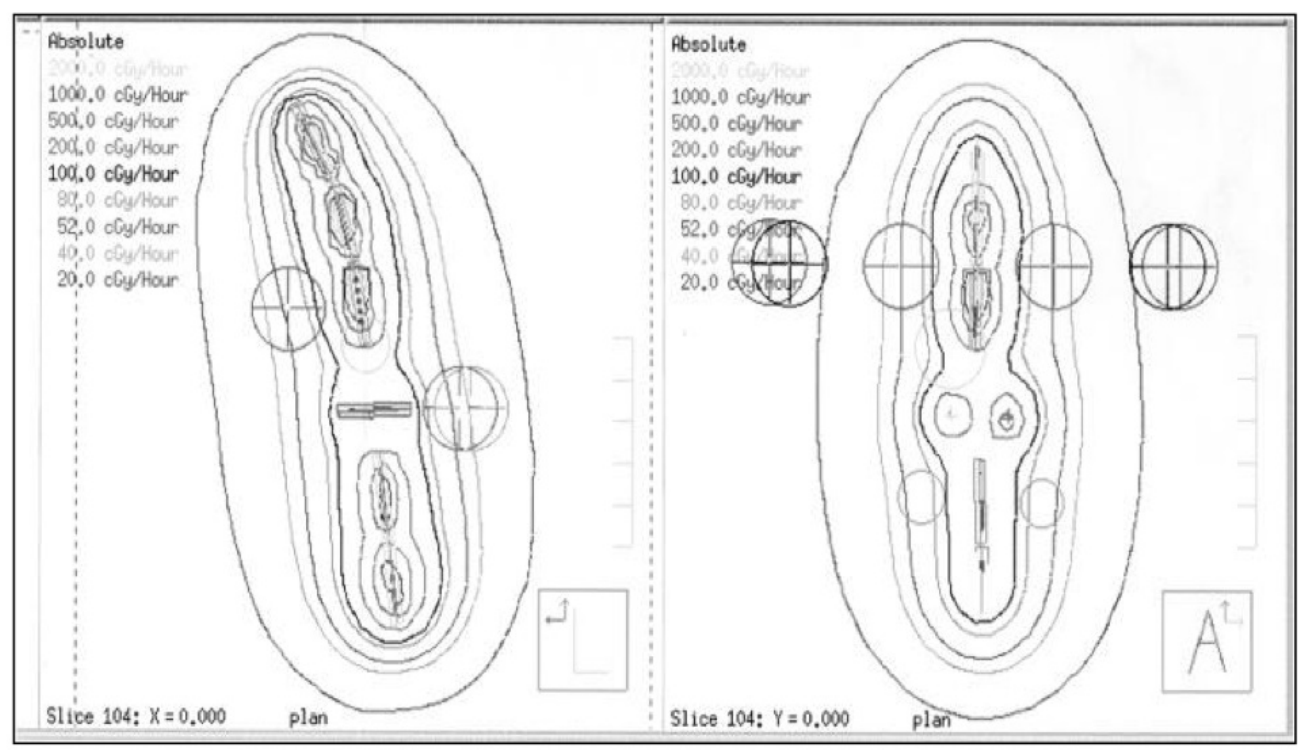

FIGURE 4: Isodose distributions around loaded Gynocyte Applicator

Between November 29, 2000 and March 14, 2003, 25 patients with histologically proven carcinoma of the cervix uteri were accrued to this clinical trial. Appropriate committee approval was obtained through the local IRB [Institutional Review Board] (University of Miami IRB \#20057286). Eligibility criteria included: biopsy proven diagnosis, lack of previous use of brachytherapy and Karnofsky Performance Status of $>70$. Demographic (e.g., age and ethnicity) and tumor (e.g. stage, histology, tumor maximum diameter on examination, and extent of vaginal extension) characteristics were all recorded. Treatment was prescribed according to disease stage and only one LDR intracavitary brachytherapy application was employed in each patient. The practice at UM MSM has been to use a single insertion based on a previous publication from this institution showing an increased likelihood of completing the entire course of radiation therapy in the population served within eight weeks with one insertion versus two [2]. Patients were followed periodically for up to four years. The median duration of follow-up was 38 months (seven to 48 months). All treatment was completed within eight weeks.

Twelve patients had "minimal" vaginal extension of tumor, nine of whom required loading of vaginal sources along with "ovoid--equivalent" sources into the Gyncocyte ${ }^{\mathrm{R}}$. The primary endpoints used in the analysis were the doses delivered to: Point A, rectum, urinary bladder, and vaginal surface, all as defined by the ICRU publication No. 38 [3]. Secondary end-points were recurrence or distance metastases, survival, and occurrence of adverse events using the National Cancer Institute, Common Terminology Criteria for reporting Adverse Events (NCI, CTCAE), version 3.0 [4]. In particular, vaginal stenosis is graded in the CTCAE as follows: Grade 1 (G1) is any vaginal narrowing and/or shortening that does not inhibit function; Grade 2 (G2) is G1, plus interference with function; and Grade 3 (G3) is defined as complete truncation of the vaginal canal that is not surgically correctable.

Twenty-four out of the 25 total patients in this study underwent external beam radiotherapy (EBRT) and 


\section{Cureus}

concurrent cisplatin weekly IV infusion $\left(40 \mathrm{mg} / \mathrm{m}^{2}\right)$ for six weeks with most (19 out of 24 patients) receiving all six cycles of chemotherapy (range: four to six cycles). No study patient required a dose deescalation of drug. Cesium-137 sources were used to deliver a mean dose rate of $0.582 \mathrm{~Gy} / \mathrm{hr}$. One patient had cervical carcinoma in-situ and received only intracavitary brachytherapy to the cervix and vagina.

Summary statistics were calculated for each end-point and a coefficient of variation (COV) of dose at each of the specified points was calculated (COV= Standard deviation X 100 /sample mean). The COV was used as a relative measure of dispersion of values around the dose prescribed or set at a specific point (i.e., a measure of accuracy). Incidence of vaginal stenosis, rectovaginal (RV) fistula, and proctitis were reported. Rates of LR recurrence and distant metastases were calculated by the method of Cumulative Incidence treating these events and death with no evidence of disease as competing risks [5]. Kaplan-Meier method [6] was used to calculate recurrence-free and overall survival data. An attempt was undertaken to analyze the relationship between the incidence of adverse events and the activity of the vaginal tandem sources. The pre-insertion operator-defined limit on acceptable dose variability at each specified point was derived from criteria established by one of this study's investigators (A.W.) that this individual has been using since July 1989 in the management of patients with cervical cancer undergoing definitive radiation therapy using both EBRT and LDR intracavitary Cesium 137 brachytherapy. Specifically, a maximum 10\% deviation from the prescribed dose (or 10\% above tolerance dose) at any defined point of interest was permitted. Thus, the prescribed cumulative total dose to point A was targeted at $85 \mathrm{~Gy}$ (minimum of $76.5 \mathrm{~Gy}$, maximum of $93.5 \mathrm{~Gy}$ ). The rectal point dose and urinary bladder point dose limits were set at 70Gy (maximum of $77 \mathrm{~Gy}$ ) and $75 \mathrm{~Gy}$ (maximum of $82.5 \mathrm{~Gy}$ ), respectively. Moreover, the upper two-thirds of the vaginal mucosa dose limit was established at $120 \mathrm{~Gy}$ (maximum $132 \mathrm{~Gy}$ ), while the lower one-third of the vaginal surface dose limit was $95 \mathrm{~Gy}$ (maximum 104.5 Gy). All of the aforementioned doses represent simple sums of doses delivered by EBRT and by intracavitary radiotherapy (ICRT) without correction for any radiobiological differences between the two modalities of treatment.

\section{Results}

The patient and disease characteristics of the studied cohort are summarized in Table 1 . It is noteworthy that $76 \%(19 / 25)$ of patients had FlGO clinical IIB or greater stage and a median tumor size of $6 \mathrm{~cm}$. Median follow-up for all study patients was 38 months.

\section{Age at diagnosis}

30 to 39

40 to 49

median 54 , range 33 to 65

Race/ethnicity

Black

Hispanic

Histology

Squamous cell 


\section{Cureus}

Carcinoma (NOS)

FIGO stage

0 (in situ)

IB2

II (1 II A, 81IB)

9

36

III (1111 A, 8 IIIB)

9

36

IVA

Tumor diameter (cm)

0 (in situ)

$4-5 \mathrm{~cm}$

6-7 cm

6

$8+\mathrm{cm}$

median 6 , range 4 to 10 , excluding in situ \&

missing

Tumor extension to Vagina

None

Upper

5

Table 2 summarizes the primary end-points for dosimetry used in the analysis. All 25 patients are included in the calculated figures in this table. One patient (with carcinoma-in-situ) was treated with ICRT alone, while 24 patients each received both EBRT with concurrently chemotherapy that was then followed by ICRT. For these latter 24 patients, the median whole pelvic EBRT dose to both points A and B was $45 \mathrm{~Gy}$ (39.59-45 Gy) delivered with megavoltage irradiation at $1.8 \mathrm{~Gy}$ per fraction. This was followed by a parametrial external beam boost at $1.8 \mathrm{~Gy}$ per fraction bringing the total dose at point B to $54 \mathrm{~Gy}$. One patient, who had had clinical Stage I B2 disease with biopsy-proven para-aortic nodal metastasis also, received $45 \mathrm{~Gy}$ at $1.8 \mathrm{~Gy} /$ fraction to the para-aortic region using an extended four-field "box" technique. 


\section{Cureus}

\begin{tabular}{|c|c|c|c|c|c|c|}
\hline & Median & Mean & SD & Minimum & Maximal & co v \\
\hline & ffill & ffill & ffill & ffill & ffill & CL) \\
\hline Point A & 84.76 & 83.86 & 2.21 & 78.18 & 88.69 & 2.6 \\
\hline Rectum & 76.56 & 69.77 & 5.69 & 50.74 & 76.5 & 8.15 \\
\hline Bladder & 75.33 & 74.26 & 4.51 & 66.55 & 80.58 & 6.07 \\
\hline Vaginal & 95.37 & 96.57 & 9.17 & 82.91 & 113.65 & 9.5 \\
\hline
\end{tabular}

\section{TABLE 2: Dosi metric End-Points: Total Doses Delivered}

$\mathrm{SD}=$ Standard Deviation; $\mathrm{COV}=$ Coefficient of Variation

It should be noted from Table 2 that no study patient exceeded the maximum allowed dose to the normal tissues (rectum, bladder, and vagina) of $110 \%$. Because of this variability, albeit small, the dose delivered through the ICRT application to supplement the total dose to point A also varied. For all 25 subjects, the median cumulative dose to point A was $84.76 \mathrm{~Gy}$ that ranged from $78.18 \mathrm{~Gy}$ to $88.69 \mathrm{~Gy}$. Figure 5 is a scatter-gram of the total dose to point $\mathrm{A}$ in relation to a prescribed dose of $85 \mathrm{~Gy}$.

\section{Total dose to point A (cGY)}

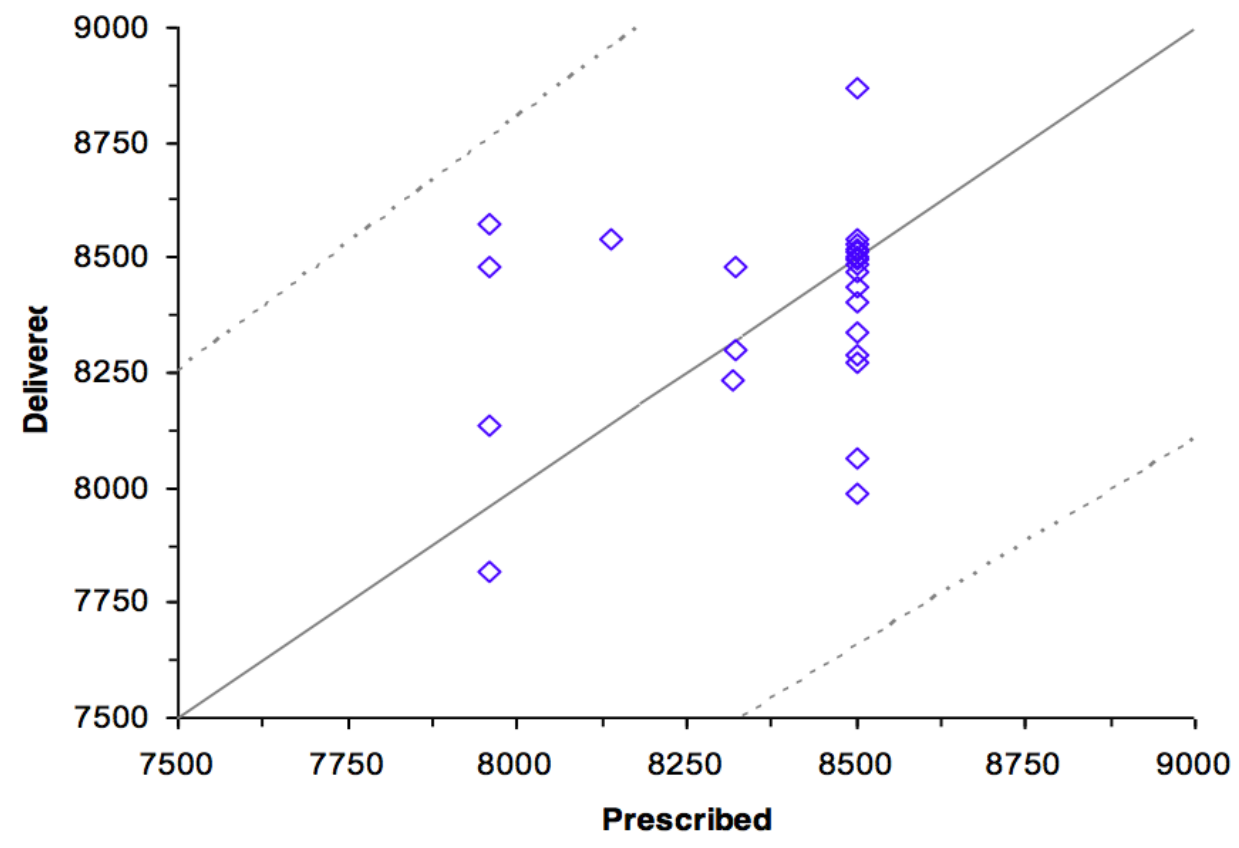

Not show $\mathrm{n}: \mathrm{PDD}=6500, \mathrm{CDD}=6522$, one patient $\mathrm{w}$ ith in situ disease

FIGURE 5: Total calculated dose delivered to point $A$ in relation to prescribed dose (24 patients with invasive cancer)

Secondary end-points: recurrence, survival and adverse events 


\section{Cureus}

Table 3 summarizes follow-up for recurrence and survival.

\section{Follow up (months)}

Recurrence

Number Median Range

(Patients)

Local

Distant

None

7

17

Vital status

Died* $^{*}$

Alive

21
27.2

17.5

$6.9-38.4$

48

$12.9-48.0$

13.7

$8.7-19.6$

48

$11.8-48.0$

TABLE 3: Fate of 25 Patients treated

One patient had local failure after 27.2 months, and seven developed distant metastases with a median follow up of 17.5 months (range 6.9 to 38.4 months). Kaplan-Meier estimates for the proportion of patients alive and recurrence-free (no evidence of LR disease or distant metastases) at one and three years were 88\% (95\% Confidence Interval (Cl): 67.3 to $96.0 \%$ ) and $65.6 \%$ (95\% Cl: 42.5 to 81.2\%), respectively. The three year cumulative incidence rates for LR relapse, distant failure, and death from other causes were $4.7 \%$ (95\% CI: 0.7 to $31.2 \%$ ), $25.6 \%$ (95\% CI: 12.7 to $51.3 \%$ ), and $4.2 \%$ (95\% CI: 0.6 to $28.5 \%)$, respectively. There was one death due to cervix cancer that occurred after 11.6 months of entry on study. Four patients died after a median follow-up of 13.7 month (range 8.7 to 19.6 months), while 21 patients remained alive after a median follow-up of 48 months (range 11.8 to 48 months). Kaplan-Meier estimates of one- and two year survival were $92.0 \%$ (95\% Cl: 71.6 to $97.9 \%$ ) and $82.8 \%$ (95\% Cl: 60.1 to 93.2\%), respectively. These findings are displayed in Figures 6-8 respectively.

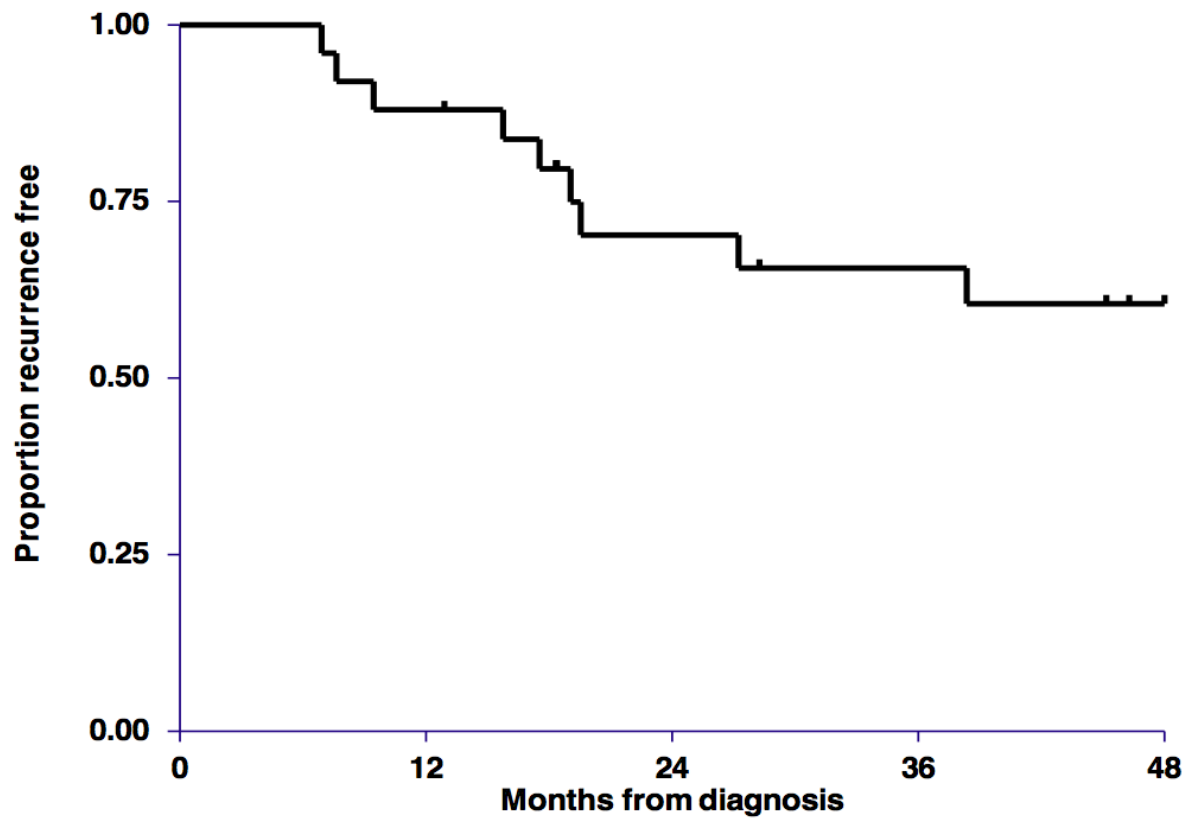




\section{Cureus}

FIGURE 6: Recurrence-Free Survival interval for 25 treated patients

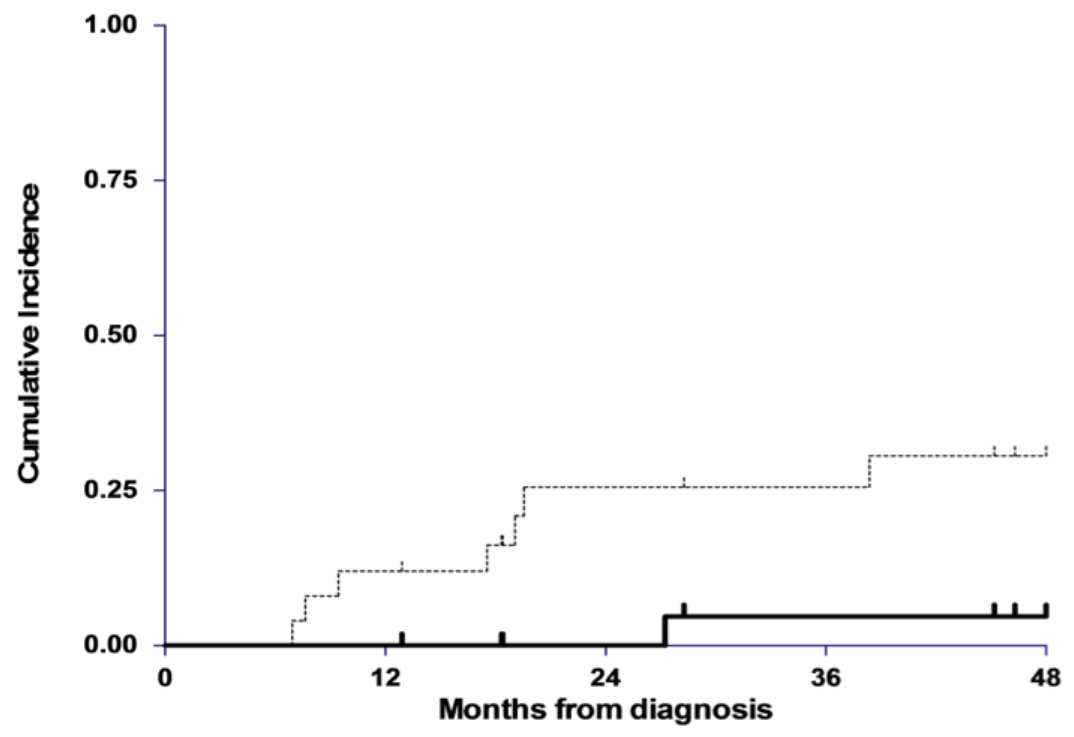

FIGURE 7: Cumulative Incidence of loco-regional and distant failure for 25 treated patients

[Loco-regional failure, solid line; Distant metastasis, dashed line; Death from other cause (competing risk), not shown]

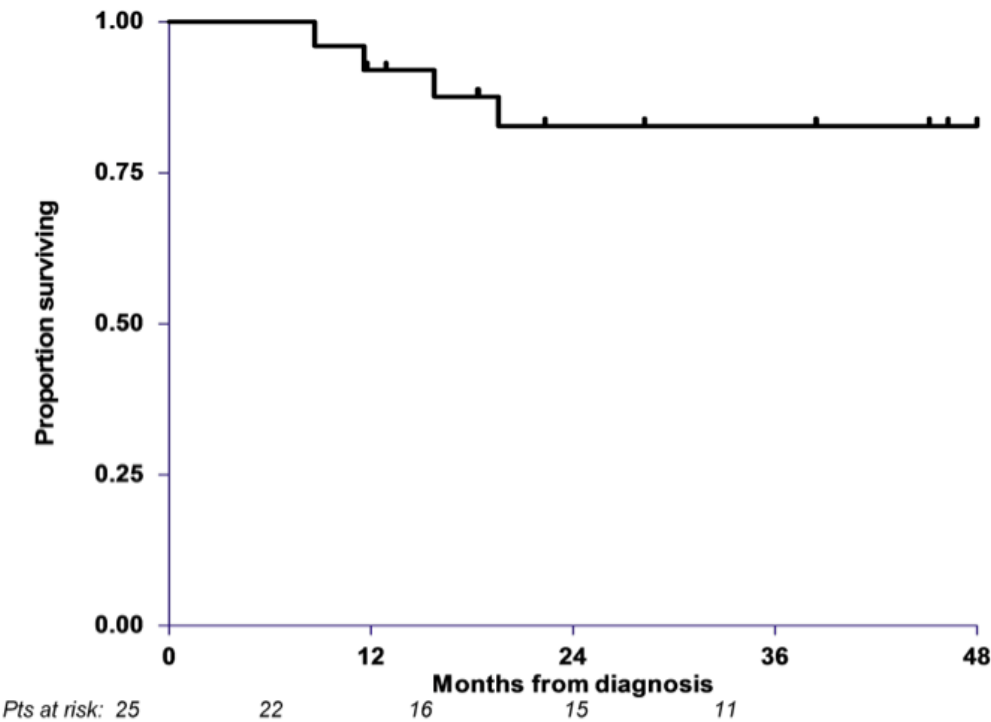

FIGURE 8: Overall Survival for 25 treated patients 


\section{Cureus}

\begin{tabular}{|l|l|l}
\hline Event & Incidence & Comment \\
\hline Vaginal Stenosis & $2 / 25(20 \%)$ & All G2 \\
G2 Proctitis & $6 / 25(24 \%)$ & All resolved with treatment \\
G3 Proctitis & $1 / 25(4 \%)$ & Resolved with treatment \\
Rectovagi nal & $3 / 25(12 \%)$ & 2 resolved with treatment (surgical treatment in one and medical treatment in the other) \\
Fistula & None & None \\
Symptomatic & None & None \\
Cystitis & None & None
\end{tabular}

TABLE 4: Adverse Events Recorded in patients on study (NCI-CTCAE version 3.0)

$\mathrm{G}=$ Grade; $\mathrm{NCI}=$ National Cancer Institute; $\mathrm{CTCAE}=$ Common Terminology Criteria for reporting Adverse Events

No hemorrhage, ulceration or Grade 3 mucositis were noted. There were three patients (12\%) that developed RV fistulae; however, the specific location of each could not be determined from the available medical records. Table 5 summarizes their disease characteristics and outcome.

\begin{tabular}{|l|l|l|l|l|l|}
\hline $\begin{array}{l}\text { FIGO } \\
\text { Stage }\end{array}$ & $\begin{array}{l}\text { Loading of Vaginal Portion of } \\
\text { Tandem }\end{array}$ & $\begin{array}{l}\text { Total Rectal Length } \\
\text { in in. }\end{array}$ & $\begin{array}{l}\text { ICRU Total Rectal } \\
\text { Dose }\end{array}$ & $\begin{array}{l}\text { Max Total Rectal } \\
\text { Dose }\end{array}$ & Status \\
\hline IIA & yes & 6 & 70.92 & 77.51 & Alive/NED \\
IIIA & yes & 8 & 75.27 & 86.28 & Alive/NED \\
1B2 & no & 2 & 72.56 & 72.56 & Dead/Liver \\
\end{tabular}

TABLE 5: Characteristics of patients developi ng rectovagi nal fistulae.

ICRU = International Commission on Radiation Units and Measurements; NED = No Evidence of Disease

Two of the three patients had vaginal sources loaded into the applicator along with the "ovoidequivalent" sources. One died of liver metastases. In the other two patients, the fistulae required surgical management in one and healed by conservative medical measures in the other. Figure 9 is a scatter-gram that demonstrates that none of the studied patients, including those developing RV fistulae, received more than the $110 \%$ of the allowable cumulative dose to the bladder and rectum (ICRU point). 


\section{Cureus}

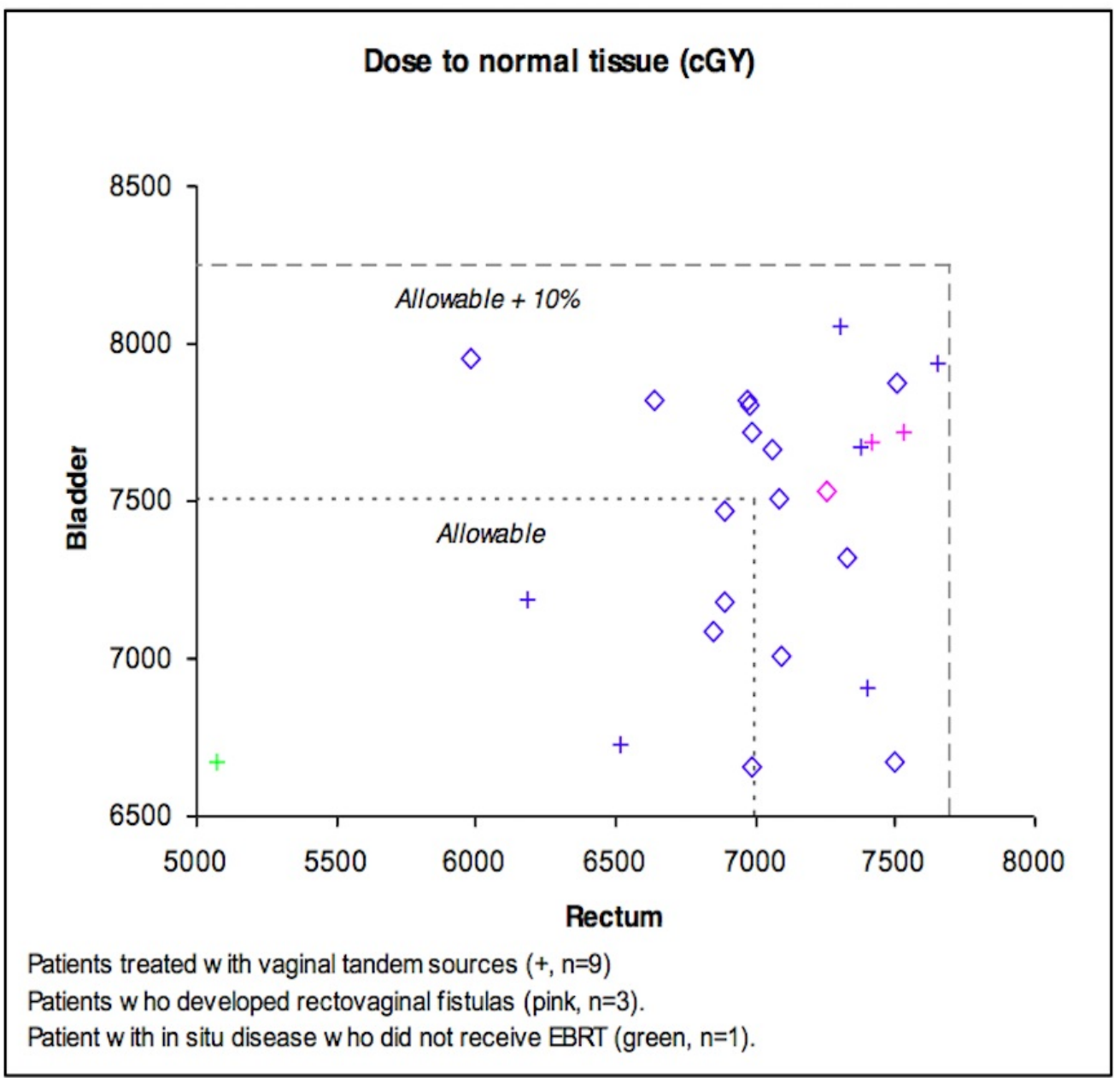

\section{FIGURE 9: Cumulative radiation dose to normal tissues, 25 treated patients, and development of rectovaginal fistulae}

All three of these patients with RV fistulae were Iocally controlled at the time of last contact. The sample size precluded conclusive analyses with respect to the correlation of the development of recto-vaginal fistula and treatment variables. In addition, CT dosimetric planning was not performed as part of this study; thus, a volumetric analysis concerning the rectal dose to the entire anterior rectal wall could not be determined.

\section{Discussion}

In the U.S., LDR brachytherapy continues to play a significant role in the management of cervical cancer. According to the Patterns of Care Studies [7-8], 92.4\% of patients treated with non-palliative intent received brachytherapy, of which $78 \%$ received LDR intracavitary and 5.5\% received LDR interstitial treatment. Similar figures had been reported earlier in the American Brachytherapy Society Survey [9]. Although there has been a recent increase in the use of high dose-rate (HDR) brachytherapy, indicating a possible shift of such pattern of care, the biological equivalence of doses delivered by either dose rate in comparison to each other remains subject to controversy as emphasized in recent reports [10-11].

With respect to the primary end-points of the study, the results show that the device can deliver the prescribed doses with acceptable reproducibility and accuracy. As one may expect, technical accuracy of intracavitary insertions can influence pelvic control and survival as noted by Corn, et al. [12]. In their series of 128 patients who were treated with EBRT and the intracavitary Fletcher-Suit-Delclos (FSD) LDR system of tandem and ovoids, these investigators reported on multivariate analysis that technical adequacy of the brachytherapy implant was the most important prognostic disease discriminant of local 
control. Katz and Eifel [13] quantified intracavitary brachytherapy parameters of 808 applications of the FSD system in 396 patients and reported remarkable geometric consistency of applicator placement using the guidelines described by Fletcher [14].

Although doses at reference points were not relied upon in the treatment planning process of their patients, optimized applicator geometry resulted in narrow ranges in the doses delivered to the reference points with median total doses of 87 (83-88) Gy, 68 (65-74) Gy and 70 (64-76) Gy to point A, rectum and bladder ICRU-defined reference points, respectively. Nevertheless, Delclos, et al. [15] in an earlier publication had commented on the difficulty of maintaining the colpostats in the lateral fornices. This was especially true when the posterior fornix was spacious, as the colpostats had a tendency to fall into the posterior forniceal spaces, which lead to a modification of the system with the addition of a "yoke" for better stabilization. The applicator used in our study is designed to minimize such problems due to its fixed structure. Although not addressed in this study, the MRI-compatibility of the plastic alloy may be of future potential interest given the trends in treatment planning and optimization of treatment of cervical cancer using MRI and may, perhaps, offer an added benefit to its utility in future studies.

Although the incidence and mortality of cervical cancer are comparatively low in the developed world, among women worldwide, cervical cancer remains the second most common type of cancer [16]. The International Agency for Research on Cancer estimates that cervical cancer was responsible for approximately 250,000 deaths in 2005, ranking as the third leading cause of cancer deaths in women worldwide [17-18]. With the increasing sophistication of the technology of intracavitary radiation delivery that uses 3-dimensional (D) imaging to conform dose to target volumes, while sparing organs at risk [19-20], it should be borne in mind that the majority of patients with cervical cancer around the world do not have ready access to such radiographic innovations. Simplicity of use of the applicator described in this study may be advantageous in such context.

Despite the relatively high proportion of locally advanced disease in the study population FIGO stage and bulk of disease as shown in Table 1, the use of the applicator did not adversely affect loco-regional control (24 out of 25 without LR relapse) in the cohort studied. In fact, our data in this regard are comparable with those of much larger series where patients were treated with chemo-radiotherapy as analyzed recently by Green, et al. [21-22]. By way of example, for early-stage and locally advanced disease, the five-year rates of central pelvic disease control were $94 \%$ and $87 \%$, respectively, in the analysis by Katz and Eifel [13]. However, the small sample size of our cohort is acknowledged as a difficulty inherent to pilot studies rendering it impossible to draw firm conclusions.

Except for the incidence of RV fistulas, the rates of adverse events reported in our series are comparable to those of other series $[13,23]$. In a review of the patient database at one prominent institution with longstanding experience in the use of definitive radiotherapy incorporating both external beam irradiation and LDR ICRT and predominantly without concurrent chemotherapy, the proportion of RV fistulae according to clinical stage of disease was as follows: I B - 4/493 (0.8\%); I IA- 2/151 (1\%); II B -11/433 (2\%); III - 11/350 (3\%); and IVA - 1/29 (3\%) [24]. Attempts at correlating clinical complications reported in our study to ICRU-defined rectal and bladder points may be fraught with difficulties. The reason for this is that these points may not be representative of maximal organ dose as shown by numerous studies utilizing 3D imaging [25-27]. Although the exact location of the RV fistulae in this current study could not be identified, one of these CT-based dosimetric planning series demonstrated that the maximal rectal dose from ICRT was "found at a point superior to the vaginal fornix" [26].

For the three patients in this present study who developed RV fistula, the planning intraoperative orthogonal radiographs were retrospectively re-evaluated by one individual from our Investigational Team (X.W.). As depicted in Table 5, it became apparent that in two cases there was a considerable disparity regarding the ICRU rectal dose determination and the maximum total rectal dose (that occurred within $3 \mathrm{~cm}$ inferior to the "ovoid--equivalent" sources) when the vaginal portion of the Gynocyte ${ }^{\mathrm{R}}$ was loaded with Cesium 137 sources.

Alternatives methods, to ICRU rectal points, to be used for estimating rectal dose have indeed been suggested for better prediction of rectal complications [28-29]. Stryker, et al. [30] showed a significant correlation of brachytherapy dose to points "Rl" (on the anterior rectal wall at the level of the posterior fornix) and "R2" (the site where the recto-sigmoid is closest to the tandem) with severe rectal injuries. 
Cheng, et al. [25] found that the difference between patients with and without late rectal complications was more prominent for the "maximal proximal rectal dose" (described as the point of highest dose in a 5 -cm interval spacing from the anal verge for four to five points proximally) than for ICRU rectal dose in 75 patients who received intracavitary HDR brachytherapy and EBRT. Recommendations for 3D dosevolume parameters for 3D image-based treatment planning of brachytherapy in cervix cancer have been formulated by the (GYN) GEC ESTRO working group [20]. Such recommendations, it should be pointed out, were published after our study had been concluded. The expectation is that such treatment planning concepts will replace the traditional ones after a transitional period.

In addition, the fact that gauze packing at the vaginal apex (mandatory with available applicators) could not be used with the version of the Gynocyte ${ }^{\mathrm{R}}$ employed in this study must also be considered as a contributing factor to the higher than expected rate of RV fistulae (12\%). The use of vaginal packing as a pivotal element of minimizing dose to both bladder and rectum was shown by Katz and Eifel [13]. One requirement for proper placement of LDR intracavitary applicator, cited by these two authors, was the demonstration of maximum displacement of the radio-opaque vaginal packing from a line extending caudad from the posterior edge of the ovoids parallel to the tandem, on the lateral films. In $92 \%$ of their patients, the vaginal packing "displaced the rectum within $5 \mathrm{~mm}$ or outside a vertical line dropped from the back of the ovoids parallel to the axis of the tandem" [13].

Based on our current quantitative review of the patients who developed RV fistulae and prior to any current widespread commercial distribution of the Gynocyte ${ }^{\mathrm{R}}$, a new design modification to the posterior aspect of the device is being implemented to simulate the gauze posterior vaginal packing described for standard LDR systems. Specifically, a plastic pouch will line the length and width of the posterior aspect of the cylinder that can be differentially inflated with a mixture of saline and contrast material to achieve a $5 \mathrm{~mm}$ expansion of the anterior rectal wall away from the posterior surface of the applicator. This will allow rectal dose optimization whenever Cesium 137 sources are loaded into the vaginal component along with source placement into the "ovoid-equivalent" receptacles.

As a final consideration, it must also be borne in mind that those patients who experienced RV fistulae also received a total radiation dose to point A of approximately $85 \mathrm{~Gy}$ concurrently with cisplatin. In one report [31], there was an increase in chronic grade 3 and 4 gastrointestinal toxicities when chemotherapy was given concurrently with radiotherapy to total doses of at least $85 \mathrm{~Gy}$ to Point A as compared with lower total doses of around $81 \mathrm{~Gy}$.

\section{Conclusions}

In conclusion, these data support the hypothesis of uniform dose delivery to point A, rectum, bladder and vaginal surface by this novel applicator with a high degree of accuracy and precision without compromising the anticipated rate of LR control. The limitation imposed by the sample size of this pilot study is acknowledged. The results should stimulate interest in further investigation of this novel LDR brachytherapy applicator for patients with cancer of the cervix, especially in the presence of' minimal" extension of tumor involving the mid and lower portions of the vagina. Due to the higher than expected incidence of RV fistulae in the present trial, more investigations are needed. In particular, studies involving 3-D volumetric analyses of the rectal doses are indicated in conjunction with the aforementioned ongoing modification of design of the device, intended to minimize anterior rectal wall dose, before its widespread clinical application can be advocated.

\section{Additional Information \\ Disclosures}

Human subjects: Consent was obtained by all participants in this study. University of Miami issued approval \# 20057286. Animal subjects: All authors have confirmed that this study did not involve animal subjects or tissue. Conflicts of interest: In compliance with the ICMJE uniform disclosure form, all authors declare the following: Payment/services info: All authors have declared that no financial support was received from any organization for the submitted work. Financial relationships: All authors have declared that they have no financial relationships at present or within the previous three years with any organizations that might have an interest in the submitted work. Intellectual property info: Since July 2004, Drs. Wolfson and Wu have had a signed commercial licensing agreement with Bio- 
Nucleonics, Inc. that was arranged by the University Of Miami Office Of Technology which allows BioNucleonics to commercially manufacture and distribute this device. Drs. Wolfson and Wu receive royalty payments from this licensing agreement. Other relationships: All authors have declared that there are no other relationships or activities that could appear to have influenced the submitted work.

\section{Acknowledgements}

Authors Contributions: All authors contributed equally to manuscript.

\section{References}

1. Wolfson A H, Wu X, Takita C, et al.: A novel applicator for low-dose rate brachytherapy of gynecological cancers. Int J Gynecol Cancer. 2003, 13:532-540. 10.1046/j.1525-1438.2003.13012.x

2. Abdel-Wahab M, Wolfson A, Ossi P, et al.: University of Miami experience using one versus two intracavitary brachytherapy implants. Am J Clin Oncol (CCT). 2002, 25:313- 317.

3. International Commission on Radiation Units and Measurements Dose and Volume Specification for reporting intracavitary therapy in Gynecology. 1985, 38:

4. National Cancer Institute, Common Terminology Criteria for Adverse Events, Version 3.0 . (Unknown). Accessed: December 21, 2005:

https://webapps.ctep.nci.nih.gov/webobjs/ctc/webhelp/Welcome_to_CTCAE.htm\#Welcome_to_CTCAE_1.h

5. Marubini E, Valsecchi M: Analysing Survival Data from Clinical Trials and Observational Studies . John Wiley, New York, New York; 1996.

6. Kaplan E, Meier P: Nonparametric estimation from incomplete observations . Journal of the American Statistical Association. 1958, 53:457-481. 10.1080/01621459.1958.10501452

7. Eifel P, Moughan J, Erickson B, et al.: Patterns of radiotherapy practice for patients with carcinoma of the uterine cervix: a pattern of care study. Int J of Radiat Oncol Biolog Phys. 2004, 60:1144-1153.

8. Erickson B, Eifel P, Moughan J, et al.: Patterns of brachytherapy practice for patients with carcinoma of the cervix (1996-1999): A Patterns of Care Study. Int J Radiat Oncol Biol Phys. 2005, 63:1083-1092. 10.1016/j.ijrobp.2005.04.035

9. Nag S, Orton C, Young D, et al.: The American Brachytherapy Society survey of brachytherapy practice for carcinoma of the cervix in the United States. Gynecol Oncology. 1999, 73:111-118. 10.1006/gyno.1998.5334

10. Sminia P, Schneider C, Fowler J: The optimal fraction size in high-dose-rate brachytherapy: dependency on tissue repair kinetics and low-dose rate. Int J Radiat Oncol Biol Phys . 2002, 52:844-849. 10.1016/S0360-3016(01)02750-X

11. Urbanski K, Gasinska A, Pudelek J, et al.: Biologically effective doses in radiotherapy of cervical carcinoma. Neoplasma. 2004, 51:228-238.

12. Corn B, Hanlon A, Pak T, et al.: Technically accurate intracavitary insertions improve pelvic control and survival among patients with locally advanced carcinoma of the uterine cervix. Gynecol Oncology. 1994, 53:249-300. 10.1006/gyno.1994.1137

13. Katz A, Eiffel P: Quantification of intracavitary brachytherapy parameters and correlation with outcome in patients with carcinoma of the cervix. Int J of Radiat Oncol Biolog Phys. 2000, 48:1417-1425. 10.1016/S0360-3016(00)01364-X

14. Fletcher G: Textbook of Radiotherapy, 3rd Edition. Lea and Febiger, Philadelphia, PA; 1980.

15. Delclos L, Fletcher G, Moore E, et al.: Minicolpostats, dome cylinders, other additions and improvements of the Fletcher-Suit afterloadable system: indications and Iimitations of their use. Int J of Radiat Oncol Biolog Phys. 1980, 6:1195-1206.

16. Drain P, Holmes K, Hughes J, et al.: Determinants of cervical cancer rates in developing countries . Int J Cancer. 2002, 100:199-205. 10.1002/ijc.10453

17. Parkin D, Bray F, Ferlay J, et al.: Global Cancer Statistics, 2002 CA Cancer . J Olin. 2005, 55:74-108.

18. Ferlay J, Bray F, Pisani P, et al.: GLOBOCAN 2002. Cancer Incidence, Mortality and Prevalence Worldwide. IARC Cancer Base No. 5 Version 2.0. IARC Press, Lyon, France; 2004.

19. Nag N, Cardenes H, Chang S, et al.: Proposed guidelines for image-based intracavitary brachytherapy for cervical carcinoma: Report from Image-Guided Brachytherapy Working Group. Int J Radiat Oncol Biolog Phys. 2004, 60:1160-1172. 10.1016/j.ijrobp.2004.04.032

20. Potter R, Haie-Meder C, Van Limbergen E et al.: Recommendations from gynaecological (GYN) GEC ESTRO working group (II): Concepts and terms in 3D image-based treatment planning in cervix cancer brachytherapy-3D dose volume parameters and aspects of 3D image-based anatomy, radiation physics, and radiobiology. Radiotherapy and Oncology. 2006, 78:67-77.

21. Green J, Kirwan J, Tierney J, et al.: Survival and recurrence after concomitant chemotherapy and radiotherapy for cancer of the uterine cervix: a systematic review and meta-analysis. Lancet. 2001, 358:781-786. 10.1016/S0140-6736(01)05965-7

22. Concomitant chemotherapy and radiation therapy for cancer of the uterine cervix/Update . 2005, 


\section{Cureus}

Cochrane Database Syst Rev. 2005:CD002225.

23. A systematic review of acute and late toxicity of concomitant chemoradiation for cervical cancer . 2003, 68:217-226. 10.1016/S0167-8140(03)00197-X

24. Radiation therapy morbidity in carcinoma of the uterine cervix: Dosimetric and clinical correlation . 1999, 4:855-866.

25. Cheng J, Peng L, Chen Y, et al.: Unique role of proximal rectal dose in late rectal complications for patients with cervical cancer undergoing high-dose-rate intracavitary brachytherapy. Int J Radiat Oncol Biol Phys. 2003, 57:1010-1018.

26. Pelloski C, Palmer M, Chronowski G, et al.: Comparison between CT-based volumetric calculations and ICRU reference-point estimates of radiation doses delivered to bladder and rectum during intracavitary radiotherapy for cervical cancer. Int J Radiat Oncol Biol Phys. 2005, 62:131-137.

27. Wachter-Gerstner N, Wachter S, Reinstadler E, et al.: Bladder and rectum dose defined from MR1 based treatment planning for cervix cancer brachytherapy: comparison of dose-volume histograms for organ contours and organ wall, comparison with ICRU rectum and bladder reference point. Radiother Oncol. 2003, 68:269-276.

28. van den Bergh F, Meertens M, Moonen L, et al.: The use of a transverse CT image for the estimation of the dose given to the rectum in intracavitary brachytherapy for carcinoma of the cervix. Radioth Oncol. 1998, 47:85-90. 10.1016/S0167-8140(97)00187-4

29. Kapp K, Stuecklschweiger G, Kapp D, et al: Dosimetry of intracavitary placements for uterine and cervical carcinoma. Results of orthogonal film, TLD, and CT-assisted techniques. Radiother Oncol. 1992, 24:137-146.

30. Stryker J, Bartholomew M, Velkley D, et al.: Bladder and rectal complications following radiotherapy for cervix cancer. Gynecologic Oncology. 1988, 29:1. 10.1016/0090-8258(88)90140-0

31. Monk B, Tian C, Rose P, Lanciano, R: Which clinical/pathologic factors matter in the era of chemoradiation as treatment for locally advanced cervical carcinoma? Analysis of Two Gynecologic Oncology Group (GOG) Trials. Gynecol Oncol. 2007, 105:427-433. 